\title{
Filtration efficiency of face masks and veils as protective measures during COVID-19 pandemic
}

\author{
Khaled S. Al-Hadyan ${ }^{1, *}$, Ghazi A. Alsbeih ${ }^{1}$, Najla M. Al-Harbi ${ }^{1}$, Sara S. Bin Judia ${ }^{1}$, Maha M. Al- \\ Ghamdi ${ }^{1}$, Salem M. Al-Ghamdi², Belal A. Moftah ${ }^{1}$, Rashed S. Alhmaid ${ }^{3}$ \\ ${ }^{1}$ Dept. of Biomedical Physics, King Faisal Specialist Hospital and Research Centre \\ (KFSH\&RC), Riyadh, Saudi Arabia \\ ${ }^{2}$ Infection Control and Hospital Epidemiology Department, KFSH\&RC. Riyadh, Saudi Arabia \\ ${ }^{3}$ General Corporate Consultancy Department, KFSH\&RC, Riyadh, Saudi Arabia \\ * Correspondence authors: khadyan@kfshrc.edu.sa
}

\begin{abstract}
Wearing face masks have been implemented as a public and personal health control measure against the spread of coronavirus disease (COVID-19). However, the protection level of nonmedical face masks, such as women face veils, is still uncertain. This study aimed to assess the filtration efficiency (FE; percentage of particles retained by a mask) of different types of medical masks (either as sealed or unsealed, single or doubled), non-medical masks (cloth masks) and face veils. FE of face masks was evaluated using an in-house 3D-printed air duct connected to the Aerotrak particle counter with a capability of counting particle sizes of $0.3,0.5,0.7,1,2$ and $5 \mu \mathrm{m}$. A set of 10 earloop surgical masks, 10 tie-on surgical masks, 3 triple-layers reusable cloth masks and 3 types (short, medium and long) of traditional face veils were included in the study. The unsealed surgical masks showed intermediate FE (36.54-80.58\%), with no observed differences between tie-on and earloop or single and doubled masks. For each mask type, the mean FE values of sealed surgical masks ( $\mathrm{FE} \geq 99.16 \%)$ was significantly higher $(\mathrm{P}<0.001)$ than the unsealed ones ( $\mathrm{FE} \leq 80.58 \%$ ). No significant difference was observed in the mean $\mathrm{FE}$ values between unsealed surgical masks and either cloth masks $(\mathrm{FE}=23.19-75.35 \%, \mathrm{P}=0.26)$ or face veils $(\mathrm{FE}=19.10$ $70.68 \%, \mathrm{P}=0.14)$. However, a mockup experiment showed that wearing a surgical mask under the face veil significantly improve the FE $(33.73-79.18 \% ; \mathrm{P}<0.001)$. We conclude that besides sealed surgical masks that ensure optimal filtration under the experimental conditions, the unsealed surgical and cloth masks and face veils showed comparable performance and acceptable protection at $5 \mu \mathrm{m}$ particle size, which is the most relevant particle size associated with COVID-19 infectious droplets. Wearing a surgical mask under the face veil significantly improves the FE compared to wearing a face veil alone.
\end{abstract}

Keywords: COVID-19; filtration efficiency; face masks; face veils; surgical masks.

\section{Introduction}

The coronavirus disease (COVID-19) caused by severe acute respiratory syndrome coronavirus 2 (SARS-CoV-2) was declared as a Public Health Emergency of International Concern (PHEIC) by the end of January 2020 and a pandemic on 11 March (World Health Organization (WHO), 2020d; 
2021a). The disease that started in China in December 2019 affected, by the end of July 2021, more than 198 million individuals around the world including 4.2 million deaths (World Health Organization (WHO), 2021b).

The transmission of SARS-CoV-2 viruses is thought to occur primarily through respiratory droplets $(>5 \mathrm{um})$ released from infected patients during coughing, sneezing or speaking, and then transmitted to another healthy person by fomite transmission (Kim et al., 2020; Nak-Jung et al., 2020; O'Dowd et al., 2020). Another important transmission mechanism is smaller aerosols (i.e., $<5 \mu \mathrm{m}$ ) which remain airborne for long time and can lead to transmission in poorly ventilated environments (Sosnowski, 2021; Tang et al., 2021). Therefore, wearing disposable surgical masks, in addition to other preventive measures such as hand hygiene and social distancing, have been strongly recommended by World Health Organization (WHO) to be worn in public places to prevent the spread of SARS-CoV-2 transmission (World Health Organization (WHO), 2020c). Consequently, wearing surgical masks was implemented in more than 100 countries as one of the mandatory general precautionary measures in public places, public transportation and indoor spaces (Bussemaker, 2020). As a result, a global shortage of surgical masks occurred, evoking using reusable cloth (textile) face masks in non-medical settings (Ji et al., 2020). The surgical and cloth masks' efficacy has been extensively studied in terms of filtration efficiency, reusability and material stability (Roberge, 2008; European Centre for Disease Prevention and Control, 2020; Szarpak et al., 2020; Zangmeister et al., 2020). However, there is limited guidance and experimental data comparing the protection degree between tie-on and earloop surgical masks, single and double surgical masks and sealed and unsealed surgical masks (Huang \& Huang, 2007; Grinshpun et al., 2009; Sanchez, 2010)

Furthermore, there is a lack of data regarding the protection level of other face mask alternatives used in certain societies such as face veils. In general, face veils are ornamental or functional dresses, used more often by women than men, in certain societies for variable purposes and different beliefs. The face veils are made from either silk, cotton, chiffon or a mixture of these materials. A number of scientific-social debates were raised in the media about whether face veils provide adequate protection as ordinary face masks, with no experimental data available to settle this debate (Caldera, 2020; Perolini, 2020). Only two studies reported that face veils were used in certain countries as an alternative to the face mask during the COVID-19 pandemic (Noushad \& Al-Saqqaf, 2021; Raja et al., 2021). However, no experimental data was reported to support this assumption. $\mathrm{v}$

The current study aimed to assess the filtration efficiency (FE) of different types of medical masks (tie-on and earloop surgical masks) either as sealed and unsealed masks or as single or double masks. Furthermore, the study evaluated the FE of non-medical masks such as cloth masks and face veils.

\section{Materials and Methods}

\subsection{Medical and non-medical masks}

The description of the face masks and veils used in the study is presented in Table 1 . Briefly, 2 types of fiber medical masks (earloop and tie-on masks) and non-medical mask (cloth masks), and face veils were used. 
Table 1: Description of the face masks and veils used in this study.

\begin{tabular}{ccccc}
\hline Face mask & Type & Company & Country & Lot number \\
\hline Medical: & & & & \\
Earloop mask & Surgical mask & Enayah & Saudi Arabia & 6281141017551 \\
Tie-on mask & Surgical mask & Damad & Saudi Arabia & 322004W16 \\
Non-medical: & & KFSH\&RC & Saudi Arabia & \multirow{2}{*}{ NA } \\
$\begin{array}{c}\text { Cloth mask } \\
\text { triple-layers) }\end{array}$ & Non-Medical & Souvenir Store & & \multirow{2}{*}{ NA } \\
$\begin{array}{c}\text { Face veils (3 } \\
\text { types) }\end{array}$ & Traditional & Local handcraft & Saudi Arabia & \\
\hline
\end{tabular}

A set of 10 earloop surgical masks (Enayah), 10 tie-on surgical masks (Damad), 3 triple layers reusable cloth masks (purchased from KFSH\&RC Souvenir Store) and 9 traditional women face veils ( 3 short; from nose to chin, 3 medium; from nose to neck and 3 long; from nose to upper chest) were included in the study. The two types of surgical masks are manufactured locally, and they were selected as they are widely used in Saudi Arabia during the COVID-19 pandemic. The surgical masks' FE was measured either as single or doubled masks and either sealed or unsealed masks.

\subsection{Filtration efficiency (FE) measurement}

The FE measurement used in this study is an in-house method that was previously described (AlHadyan et al., 2021). Briefly, a custom-designed air duct was locally designed to measure the FE of medical and non-medical masks using the ambient particulate matter (PM) air as a source of the measured particles. The air duct consists of two parts, head and tail. The head part is connected to an AeroTrak particle counter (TSI, Model 9306) that counts particles with sizes of $0.3,0.5,0.7,1$, 2 and $5 \mu \mathrm{m}$ for a sampling time of 1 minute at a flow rate of 2.8 liters $/$ minute $(\mathrm{L} / \mathrm{min})$ with a storage capability of 10,000 measurements. The tail part is tied to an electrical fan that flows the PM air from the tail, all over the duct, to the head part. The fan is controlled by a variable transformer (model: 3PF1010, Staco Energy Production Company) fixed at 47 volts to give an air face velocity of $0.4 \mathrm{~m} / \mathrm{s}$; measured by Velocicalc Air Velocity Meter 9545 (TSI, product ID\# 9545-A). One measurement was carried out for each mask or veil, with a total of 104 tests for all masks and face veils used in this study.

In the case of FE assessment of sealed surgical masks, the two parts of the air duct are tied together by three mold bolts to squeeze the mask to prevent any air from sneak between both parts. For the unsealed surgical masks, cloth masks and face veils, FE was assessed to mimic their actual use where the external air can sneak into the user's face from both cheek sides. Therefore, head and tail parts were loosely connected (untied mold bolts) during the FE assessment. In addition, the FEs of face veil alone and surgical mask worn beneath face veil were assessed in a mockup experiment as they may be worn together. 
For each particle size, FE of each mask was calculated using the following formula:

$$
F E(\%)=100-\left(\frac{\text { number of penetrated particles }}{\text { average number of particles in air }} X 100\right)
$$

\subsection{Statistical analysis}

The parametric one way repeated measures analysis of variance test has been used to detect significant differences in means of different sets of data (Pervez et al., 2017; Farooq \& Freed, 2018). Therefore, it was used to detect significant differences in the FE means for each set of data obtained in this study. In case the data did not pass the normality test, the nonparametric Friedman Repeated Measures Analysis of Variance on Ranks test, which compares medians, was applied.

\section{Results}

The sealed surgical masks (for both types, earloop and tie-on), either single or doubled, showed high FE (99.16-100\%) for all particle sizes (Figure 1). However, the unsealed surgical masks, for both types, showed intermediate FE (36.54-80.58\%) either as a single ( $\mathrm{FE}=36.54-76.67 \%)$ or double masks ( $\mathrm{FE}=59.01-80.58 \%$ ). The One Way Repeated Measures Analysis of Variance test showed statistically significant differences in the mean FE values between the sealed and the unsealed surgical masks for each type (earloop or tie-on), whether single or doubled masks $(\mathrm{P}<0.001)$ (Supplementary Materials 1 and 2).

As shown in Figure 2-A, a mockup experiment showed that long face veils showed higher FE (32.02-79.83\%) than short face veils (09.03-61.62\%), medium face veils (18.24-70.59\%) and cloth masks (23.2-75.3\%). Overall, the average FE of the veils (19.1-70.7\%) was somewhat comparable to the FE of the unsealed single surgical masks (37.41-66.39\%), the unsealed tie-on surgical masks plus veil (33.73-79.18\%) and the cloth masks (23.19-75.35\%). Statistically, the results showed no significant differences in the means $\mathrm{FE}$ values between wearing a surgical mask and either a surgical mask plus (under) veils $(\mathrm{P}=0.136)$ or cloth mask $(\mathrm{P}=0.261)$ (Supplementary Material 3). However, the mean FE values of wearing a surgical mask under the veil were significantly higher $(\mathrm{P}<0.001)$ than wearing the veil alone. Overall, all masks and face veils showed better FE with increasing particle diameter (Figure 2-B). 

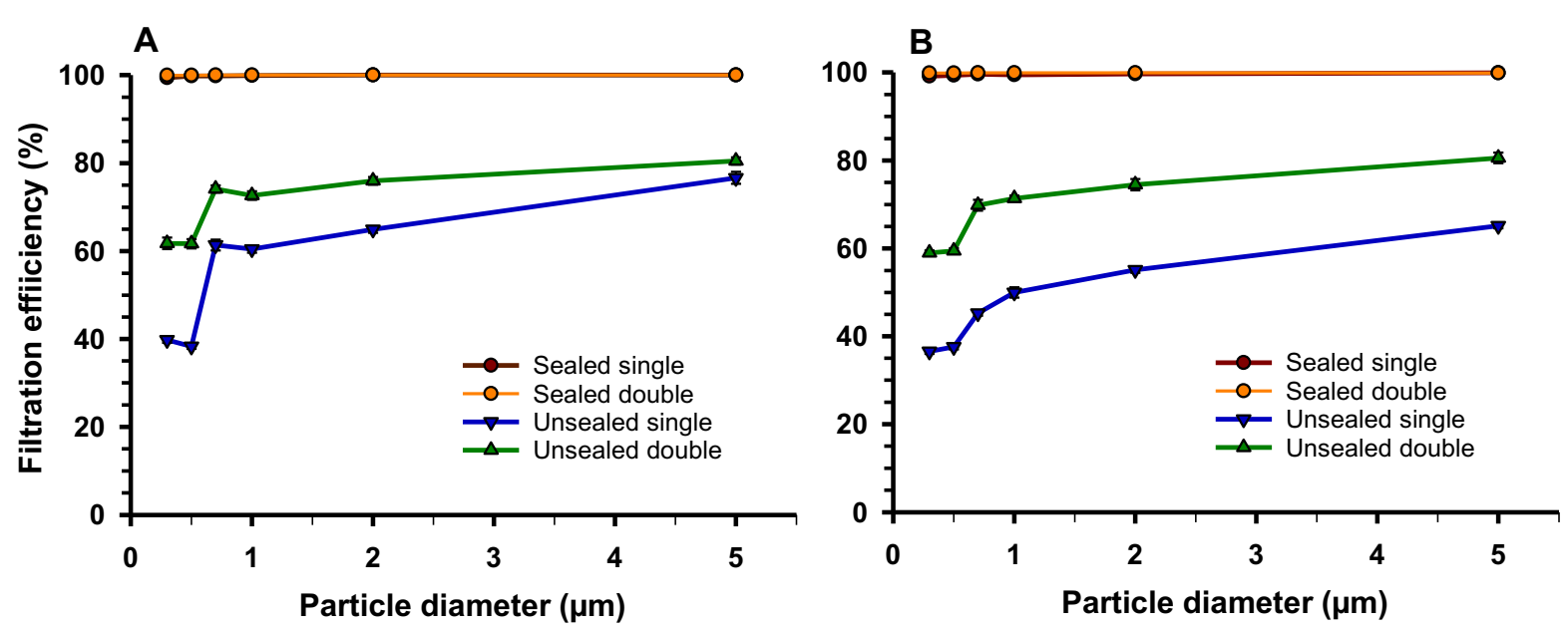

Fig. 1. Filtration efficiency (FE) of surgical earloop and tie-on surgical masks. Ten of each earloop (A) and tie-on (B) surgical masks were applied to FE evaluation for different particle sizes (0.3-5 $\mu \mathrm{m})$ using Aerotrak particle counter. The FE of the surgical masks were measured either as single or doubled masks and either as sealed or unsealed masks. Results showed significant differences in the mean FE values between the sealed and the unsealed surgical masks for both mask types (earloop or tie-on) whether single or doubled masks $(\mathrm{P}<0.001)$. The average PM concentrations (control) at $0.3,0.5,0.7,1,2$ and $5 \mu \mathrm{m}$.were 61697.3 (standard deviations 'STD'=7637.7), 4660.8 $(\mathrm{STD}=469.5), 245.5(\mathrm{STD}=22.3), 818.5(\mathrm{STD}=80.9), 864.3(\mathrm{STD}=123.7)$ and $217.3(\mathrm{STD}=$ 42.9) respectively. Symbols represent the means, while error bars, which were smaller than the symbol size, indicate the standard error.
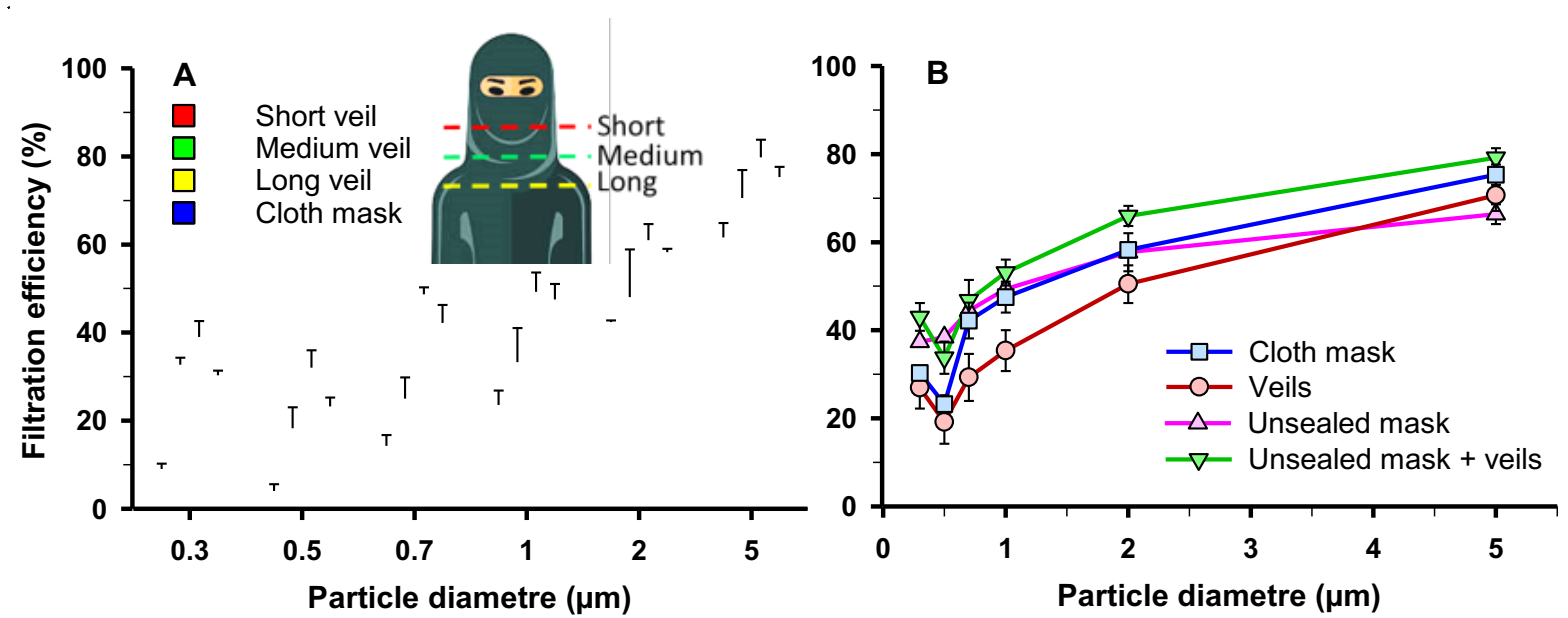

Fig. 2. Filtration efficiency (FE) of unsealed surgical masks, veil and cloth masks. A. FE of short $(n=3)$, medium $(n=3)$ and long $(n=3)$ veils and cotton mask $(n=3)$. B. FE of unsealed single tie-on masks $(n=3), F E$ average of face veils $(\mathrm{N}=9,3$ short, 3 medium and 3 long) and cotton masks $(n=3)$. All masks and face veils were applied to FE evaluation for different particle sizes (0.3-5 $\mu \mathrm{m})$. Results showed no significant differences in the means FE values between wearing a surgical mask and either a surgical mask plus (under) veils $(\mathrm{P}=0.136)$ or cloth mask $(\mathrm{P}=0.261)$. The average $\mathrm{PM}$ concentrations (control) at 0.3, 0.5, 0.7, 1, 2 and $5 \mu \mathrm{m}$.were $46530.5(\mathrm{STD}=873.3), 3257.5$ $(\mathrm{STD}=264.1), 118.2(\mathrm{STD}=14.4), 385.5(\mathrm{STD}=28.9), 374.4(\mathrm{STD}=88.0)$ and $119.0(\mathrm{STD}=38.1)$ respectively. Symbols represent the mean and error bars indicate the standard error. 


\section{Discussion}

This study evaluated the FE of medical masks (tie-on and earloop surgical masks) and non-medical masks (cloth masks and face veils). The results indicated that the unsealed surgical masks exhibited intermediate FE (36.54-80.58\%), with no observed differences between tie-on and earloop or single and doubled masks (Figure 1). For each mask type, the mean FE values of sealed surgical masks $(\mathrm{FE} \geq 99.16 \%)$ was significantly higher $(\mathrm{P}<0.001)$ than the unsealed ones $(\mathrm{FE} \leq 80.58 \%)$.In addition, no significant difference was observed in the mean FE values between unsealed surgical masks and either cloth masks $(\mathrm{FE}=23.19-75.35 \%, \mathrm{P}=0.26)$ or face veils $(\mathrm{FE}=19.10-70.68 \%$, $\mathrm{P}=0.14$ ) (Figure 2). However, wearing a surgical mask under the face veil significantly improve the FE (33.73-79.18\%; $\mathrm{P}<0.001)$. Furthermore, the in-house FE procedure used in this study is developed as an emergency alternative to the USA National Institute for Occupational Safety and Health (NIOSH) FE procedure to validate face masks during the COVID-19 pandemic (Al-Hadyan et al., 2021).

Unlike N95 Filtering Facepiece Respirators (FFRs), it is challenging to wear sealed surgical masks due to the difficulty of exporting exhaled gas, resulting in an accumulation of exhaust gas in the surgical mask and subsequently causing difficulty breathing (Huang \& Huang, 2007; Skaria \& Smaldone, 2014). Nevertheless, we have studied the FE of sealed surgical masks as healthcare frontliners have used them in case of a significant shortage of FFRs during the COVID-19 pandemic (Ekpreechakul et al., 2020; O'Kelly et al., 2020; Ekpreechakul et al., 2021).

The results indicated that sealed surgical masks, yet impractical, could give a similar FE of N95 masks by blocking at least 99.2 for all particle sizes (Figure 1). In contrast, unsealed surgical masks showed intermediate FE (36.54-80.58\%) with no observed differences between either tieon and earloop masks or single and doubled masks. Three studies support our findings by reporting comparable FE results between sealed surgical masks and N95 respirators against airborne filtration (Noti et al., 2012; Skaria \& Smaldone, 2014; Karuppasamy \& Obuchowski, 2021). In disagreement with our findings, the sealed surgical masks and cloth masks showed an average FE of $44.1 \%$ and $13.9 \%$ respectively (Joshi et al., 2020). Another study showed lower FE of the sealed surgical masks than our findings, with $\mathrm{FE}$ of $51 \%, 84 \%$ and $85 \%$ at $0.5,1$ and 2 um respectively (Sanchez, 2010). In agreement with our results, the latter study showed intermediate FE of the unsealed surgical masks, with FE of $43 \%, 75 \%$ and $79 \%$ at particle sizes of $0.5,1$ and $2 \mu \mathrm{m}$ respectively.

A unique feature of this study is the measurement of the FE of face veils worn by individual women to cover parts of their faces. The assessment of their FE is important due to the lack of data on the face veils filtration capability as compared to other cloths and medical masks. Interestingly, our findings showed that the face veils $(\mathrm{FE}=19.10-70.68 \%)$ displayed comparable $\mathrm{FE}$ to either unsealed surgical masks (37.41-66.39\%) or cloth masks (23.19-75.35\%), suggesting that face veils, surgical and cloth offer a similar degree of protection (Figure 2). Irrespective of the breathing difficulties that they may cause, our data showed that surgical mask, when worn under the veil provides a higher degree of protection than wearing a face veil alone. It was also observed that long face veil has better FE than either medium or short face veils, making veil's size is an 
important factor that affects veils' FE. Also, our data observed that cloth mask (FE=23.2-75.4\%) and unsealed surgical mask $(\mathrm{FE}=37.4-66.4 \%)$ offered comparable $\mathrm{FE}$ at particle sizes between 0.3 and $5 \mu \mathrm{m}$. In agreement with our findings, a recent report showed almost equal FE of cloth masks $(\mathrm{FE}=19.5-81.8 \%)$ and unsealed surgical masks $(\mathrm{FE}=20.8-77.3 \%)$ at particle sizes between 0.3 and $5 \mu \mathrm{m}$ (Kumar et al., 2020).

Overall, all masks and face veils showed better FE with increasing particle diameter (Figure 2-B). These differences in the FEs across different particle sizes may have an implication on the current understanding of SARS-CoV-2 transmission. The size of the SARS-CoV-2 virus is $<1.0 \mu \mathrm{m}$; however, it is transmitted through respiratory droplets $(>5 \mu \mathrm{m})$ released from COVID19 patients to another healthy person by fomite transmission (Kim et al., 2020; Nak-Jung et al., 2020; O'Dowd et al., 2020). Although droplet nuclei particles $(<5 \mu \mathrm{m})$ were not implicated to be a main transmission route of SARS-CoV-2 (World Health Organization (WHO), 2020a; 2020b; 2021a), recent reports indicated that droplet nuclei particles might play a more prominent role in SARS-CoV-2 transmission (Liu et al., 2020; Ong et al., 2020). A recent manuscript reviewed data from a number of experimental studies and theoretical models showed that the minimum size of droplets that contains and then transmit SARS-CoV-2 was $4.7 \mu \mathrm{m}$ (Lee, 2020). Therefore, the particle size of $5 \mu \mathrm{m}$ seems to be the most important size for the evaluation of the SARS-CoV-2 transmission.

Finally, it is important to note that the FE assessment in this study is based on mockup experiments to mimic the actual wearing of masks by individuals. However, other studies observed some differences in the efficiency between the mockup and actual wearing of masks (Cherrie et al., 2018; Mueller et al., 2018; Steinle et al., 2018). Further studies using appropriate custom-designed phantoms are needed to mimic actual mask performance.

\section{Conclusion}

Besides sealed surgical masks that ensure optimal filtration, the unsealed surgical mask, cloth masks and face veils showed comparable performance and acceptable protection at the $5 \mu \mathrm{m}$ particle size which is the most relevant to COVID-19 infectious droplets. Under the experimental conditions, wearing a surgical mask under the face veil significantly improves the FE compared to wearing a face veil alone.

\section{ACKNOWLEDGEMENTS}

We acknowledge King Abdulaziz City for Science and Technology (KACST) via The Fast Track Funding Path for Coronavirus Program for funding this project. (project number 5-20-01-5580005; RAC\# 2200047). We would like to thank Amr El-Sayed, Ahmad Nobah, Jeffrey Lindstrom, Sawsan Falatah, Nawarh Faran and Hanan Alsaif for their assistance and the project's administrative and financial management. 


\section{References}

Al-Hadyan, K., Alsbeih, G., Nobah, A., Lindstrom, J., Falatah, S., Faran, N., AL-Ghamdi, S., Moftah, B. \& Alhmaid, R. (2021). In-House Filtration Efficiency Assessment of Vapor Hydrogen Peroxide Decontaminated Filtering Facepiece Respirators (FFRs). International Journal of Environmental Research and Public Health, 18, 7169.

Bussemaker, C. F. A. N. (2020). Which Countries Are Requiring Face Masks? [Online]. Council on Foreign Relations. Available: https://www.cfr.org/in-brief/which-countries-are-requiring-facemasks [Accessed 02 August 2021].

Caldera, C. 2020. Fact check: Post makes faulty assertions about women and face coverings in Muslim-majority countries. USA TODAY, 19 July 2020.

Cherrie, J. W., Apsley, A., Cowie, H., Steinle, S., Mueller, W., LIN, C., Horwell, C. J., Sleeuwenhoek, A. \& Loh, M. (2018). Effectiveness of face masks used to protect Beijing residents against particulate air pollution. Occupational and environmental medicine, 75, 446-452.

Ekpreechakul, T., Saiphiroonthong, W. \& Chaiwong, W. (2021). Letter to Editor: Quantitative Fit Test of Double Layer Surgical Masks. The Bangkok Medical Journal, 17, 70-70.

Ekpreechakul, T., Saiphiroonthong, W., Chaiwong, W. \& Kunanusont, C. (2020). Quantitative Fit Tests of Selected Respirators for Health Care Workers Caring for Patients with Confirmed or Suspected COVID-19. The Bangkok Medical Journal, 16, 196-196.

European Centre For Disease PRevention And Control. (2020). Options for the decontamination and reuse of respirators in the context of the COVID-19 pandemic [Online]. Available: https:/www.ecdc.europa.eu/sites/default/files/documents/Options-for-shortage-ofsurgical-masks-and-respirators.pdf [Accessed 04 April 2021].

Farooq, M. \& Freed, S. (2018). Insecticidal activity of toxic crude proteins secreted by entomopathogenic fungi against Musca domestica L.(Diptera: Muscidae). Kuwait Journal of Science, $\mathbf{4 5}$.

Grinshpun, S. A., Haruta, H., Eninger, R. M., Reponen, T., Mckay, R. T. \& Lee, S.-A. (2009). Performance of an N95 filtering facepiece particulate respirator and a surgical mask during human breathing: two pathways for particle penetration. Journal of occupational and environmental hygiene, 6, 593-603.

Huang, J. \& Huang, V. (2007). Evaluation of the efficiency of medical masks and the creation of new medical masks. Journal of international medical research, 35, 213-223.

Ji, D., Fan, L., Li, X. \& Ramakrishna, S. (2020). Addressing the worldwide shortages of face masks. BMC materials, 2, 1-11. 
Joshi, M., Khan, A. \& Sapra, B. (2020). Quick laboratory methodology for determining the particle filtration efficiency of face masks/respirators in the wake of COVID-19 pandemic. Journal of Industrial Textiles, 1528083720975084.

Karuppasamy, K. \& Obuchowski, N. (2021). Comparison of Fit for Sealed and Loose-Fitting Surgical Masks and N95 Filtering Facepiece Respirators. Annals of work exposures and health.

Kim, J.-M., Chung, Y.-S., Jo, H. J., Lee, N.-J., Kim, M. S., Woo, S. H., Park, S., Kim, J. W., Kim, H. M. \& Han, M.-G. (2020). Identification of Coronavirus Isolated from a Patient in Korea with COVID-19. Osong public health and research perspectives, 11, 3.

Kumar, A., Bhattacharjee, B., Sangeetha, D., Subramanian, V. \& Venkatraman, B. (2020). Evaluation of filtration efficacy of various types of face-masks using ambient and PAO aerosols following with different sterilization methods. medRxiv.

Lee, B. U. (2020). Minimum sizes of respiratory particles carrying sars-cov-2 and the possibility of aerosol generation. International Journal of Environmental Research and Public Health, 17, 6960.

Liu, Y., Ning, Z., Chen, Y., Guo, M., Liu, Y., Gali, N. K., Sun, L., DuaN, Y., Cai, J. \& Westerdahl, D. (2020). Aerodynamic characteristics and RNA concentration of SARS-CoV-2 aerosol in Wuhan hospitals during COVID-19 outbreak. BioRxiv.

Mueller, W., Horwell, C. J., Apsley, A., Steinle, S., Mcpherson, S., Cherrie, J. W. \& Galea, K. S. (2018). The effectiveness of respiratory protection worn by communities to protect from volcanic ash inhalation. Part I: Filtration efficiency tests. International journal of hygiene and environmental health, 221, 967-976.

Nak-Jung, K., Chang-Kyung, K., Pyoeng-Gyun, C., Jiyoung, Y., Seong, M.-W., Jeong-Sun, S. \& Oh, M.-D. (2020). Virus Isolation from the First Patient with SARS-CoV-2 in Korea. Journal of Korean Medical Science, 84-84.

Noti, J. D., Lindsley, W. G., Blachere, F. M., CAO, G., Kashon, M. L., Thewlis, R. E., Mcmillen, C. M., King, W. P., Szalajda, J. V. \& Beezhold, D. H. (2012). Detection of infectious influenza virus in cough aerosols generated in a simulated patient examination room. Clinical Infectious Diseases, 54, 1569-1577.

Noushad, M. \& Al-Saqqaf, I. S. A. (2021). COVID-19: Is herd immunity, the only option for fragile Yemen? International Journal of Infectious Diseases.

O'Kelly, E., Arora, A., Pirog, S., Ward, J. \& Clarkson, P. J. (2020). Face Mask Fit Hacks: Improving the Fit of KN95 Masks and Surgical Masks with Fit Alteration Techniques. medRxiv.

O’Dowd, K., Nair, K. M., Forouzandeh, P., Mathew, S., Grant, J., Moran, R., Bartlett, J., Bird, J. \& Pillai, S. C. (2020). Face masks and respirators in the fight against the COVID-19 pandemic: a review of current materials, advances and future perspectives. Materials, 13, 3363. 
ong, S. W. X., Tan, Y. K., Chia, P. Y., Lee, T. H., Ng, O. T., Wong, M. S. Y. \& Marimuthu, K. (2020). Air, surface environmental, and personal protective equipment contamination by severe acute respiratory syndrome coronavirus 2 (SARS-CoV-2) from a symptomatic patient. Jama, 323, 1610-1612.

Perolini, M. (2020). Is a face mask used to fight COVID-19 really that different from a niqab? [Online]. Amnesty International. Available: https://www.amnesty.org/en/latest/news/2020/05/face-masks-and-niqabs/ [Accessed 07 April 2021].

Pervez, K., Ullah, F., Mehmood, S. \& Khattak, A. (2017). Effect of Moringa oleifera Lam. leaf aqueous extract on growth attributes and cell wall bound phenolics accumulation in maize (Zea mays L.) under drought stress. Kuwait Journal of Science, 44.

Raja, A. K. F., Raja, M. H. R. \& Ashfaq, M. (2021). The Experience and Perplexities of the COVID-19 Situation in Pakistan. International Journal of Medical Students.

Roberge, R. J. (2008). Effect of surgical masks worn concurrently over N95 filtering facepiece respirators: extended service life versus increased user burden. Journal of Public Health Management and Practice, 14, E19-E26.

Sanchez, E. (2010). Filtration efficiency of surgical masks. Master of Science, University of South Florida.

Skaria, S. D. \& Smaldone, G. C. (2014). Respiratory source control using surgical masks with nanofiber media. Annals of occupational hygiene, 58, 771-781.

Sosnowski, T. R. (2021). Inhaled aerosols: their role in COVID-19 transmission including biophysical interactions in the lungs. Current Opinion in Colloid \& Interface Science, 101451.

Steinle, S., Sleeuwenhoek, A., Mueller, W., HorwelL, C. J., Apsley, A., Davis, A., Cherrie, J. W. \& Galea, K. S. (2018). The effectiveness of respiratory protection worn by communities to protect from volcanic ash inhalation. Part II: Total inward leakage tests. International journal of hygiene and environmental health, 221, 977-984.

Szarpak, L., Smereka, J., Filipiak, K. J., Ladny, J. R. \& Jaguszewski, M. (2020). Cloth masks versus medical masks for COVID-19 protection. Cardiology journal, 27, 218-219.

Tang, J. W., Marr, L. C., LI, Y. \& Dancer, S. J. (2021). Covid-19 has redefined airborne transmission. British Medical Journal Publishing Group.

WORLD HEALTH ORGANIZATION (WHO). (2020a). Modes of transmission of virus causing COVID-19: implications for IPC precaution recommendations: scientific brief, 27 March 2020 [Online]. World Health Organization. Available: https://apps.who.int/iris/handle/10665/331601 [Accessed 02 August 2021]. 
WORLD HEALTH ORGANIZATION (WHO). (2020b). Transmission of SARS-CoV-2: implications for infection prevention precautions [Online]. Available: https://www.who.int/newsroom/commentaries/detail/transmission-of-sars-cov-2-implications-for-infection-preventionprecautions [Accessed 06 June 2021].

WORLD HEALTH ORGANIZATION (WHO). (2020c). Mask use in the context of COVID19 [Online]. Available: https:/www.who.int/publications/i/item/advice-on-the-use-of-masks-inthe-community-during-home-care-and-in-healthcare-settings-in-the-context-of-the-novelcoronavirus-(2019-ncov)-outbreak [Accessed 15 April 2021].

WORLD HEALTH ORGANIZATION (WHO). (2020d). Statement on the second meeting of the International Health Regulations (2005) Emergency Committee regarding the outbreak of novel coronavirus (2019-nCoV) [Online]. Available: https://www.who.int/news/item/30-01-2020statement-on-the-second-meeting-of-the-international-health-regulations-(2005)-emergencycommittee-regarding-the-outbreak-of-novel-coronavirus-(2019-ncov [Accessed 14 April 2021].

WORLD HEALTH ORGANIZATION (WHO). (2021a). WHO Coronavirus (COVID-19) Dashboard [Online]. Available: https://covid19.who.int/ [Accessed 31 July 2021].

WORLD HEALTH ORGANIZATION (WHO). (2021b). Coronavirus disease (COVID-19) Weekly Epidemiological Update and Weekly Operational Update [Online]. Available: https://www.who.int/emergencies/diseases/novel-coronavirus-2019/situation-reports [Accessed 13 April 2021].

Zangmeister, C. D., Radney, J. G., Vicenzi, E. P. \& Weaver, J. L. (2020). Filtration efficiencies of nanoscale aerosol by cloth mask materials used to slow the spread of SARS-CoV-2. ACS nano, 14, 9188-9200.

$\begin{array}{lr}\text { Submitted: } & 14 / 04 / 2021 \\ \text { Revised: } & 16 / 08 / 2021 \\ \text { Accepted: } & 29 / 08 / 2021 \\ \text { DOI: } 10.48129 / \text { kjs.splcov.13629 }\end{array}$

